\title{
Organización tecnológica del control de la malaria en São Paulo, Brasil, de 1930 a 19901
}

\author{
Rita Barradas Barata ${ }^{2}$
}

RESUMEN El aumento de la incidencia de malaria en varios países del mundo a partir de los años setenta, cuando parecía que estaba a punto de erradicarse la enfermedad, creó una serie de interrogantes y durante algún tiempo los malariólogos y las autoridades de salud pública escasamente comprendían lo que estaba ocurriendo. Con el fin de entender mejor este proceso, la autora del presente artículo estudió la frecuencia de casos de malaria en São Paulo, Brasil, de 1930 a 1990, procurando examinar los siguientes factores en cada una de las etapas estudiadas: la importancia social de la malaria, las condiciones en que se presentaba la enfermedad, los conocimientos epidemiológicos de la época, los instrumentos técnicos disponibles y las estrategias de control utilizadas, mediante la construcción de modelos tecnológicos. De esta manera se hizo evidente que la presentación de la enfermedad, los conocimientos sobre ella y por consiguiente las formas de hacerle frente sufrieron cambios importantes a lo largo del tiempo. Al final se discuten, a la luz de la investigación, las opciones que existen actualmente para el control de la malaria.

La presente investigación tiene un doble propósito: primero, comprender por qué aumentó la incidencia de malaria en varias partes del mundo en los años ochenta, justamente cuando se esperaba erradicar la enfermedad; segundo, buscar una explicación, aplicando un modelo teórico capaz de articular las necesidades sociales, los conocimientos epidemiológicos y las

\footnotetext{
1 Próximamente se publicará en esta revista una versión en inglés de este artículo. La investigación fue patrocinada por el Programa de Pequeñas Becas para Investigaciones en Aspectos Sociales y Económicos de las Enfermedades Tropicales del Laboratorio de Ciencias Sociales, Universidad Central de Venezuela, con el apoyo financiero del Programa Especial de Investigación y Adiestramiento sobre Enfermedades Tropicales del Banco Mundial y la OMS.

2 Facultade de Ciências Médicas da Santa Casa de São Paulo, Departamento de Medicina Social, São Paulo, Brasil. Dirección postal: Rua Cesário Motta, Jr. 61 o andar, São Paulo, SP, Brasil cep 01221-020. Fax: (055) (011) 222-0432.
}

prácticas adoptadas para satisfacer esas necesidades.

En un principio las razones propuestas para explicar el aumento de la incidencia se centraban en los problemas directamente relacionados con la forma de llevar a cabo las campañas de erradicación. Se invocaban dificultades técnicas, operativas, administrativas, financieras y políticas, a las que se atribuía el fracaso de la estrategia basada en campañas. Al hacerse, sin embargo, un análisis más detallado que articulaba dentro de un solo marco teórico la presencia de la enfermedad con sus repercusiones sociales, los conceptos epidemiológicos vigentes y las prácticas de control, saltaron a la vista nuevos aspectos que exigían explicaciones de otra naturaleza.

El objetivo central de esta investigación ha sido examinar cómo, a lo largo de distintas épocas, la malaria ha llegado a convertirse en un problema de salud de carácter social y llevado a la adopción de ciertas prácticas de control institucionales, y en qué medida los conocimientos epidemiológicos disponibles en cada época han orientado la planificación de intervenciones.

La selección del estado de São Paulo para hacer este estudio se basó en consideraciones prácticas, como la facilidad del acceso a información, y teóricas, como el hecho de que en São Paulo las instituciones dedicadas al control de la malaria son las más antiguas del país, y de que la campaña de erradicación ha progresado sin dificultades económicas, administrativas, operativas o políticas.

\section{MATERIALES Y MÉTODOS}

La investigación se basó en el análisis de documentos y los pasos metodológicos fueron los siguientes: selección 
del universo de documentos (cerca de 1500 libros, artículos, informes, pautas técnicas, etc.); lectura e indización de los documentos seleccionados (cerca de 800); separación por períodos; organización de la información en cuatro grupos temáticos (situación epidemiológica; nociones sobre la epidemiología de la enfermedad; instrumentos disponibles para su control; y organización institucional de las prácticas de intervención); elaboración de datos e identificación de los conceptos clave correspondientes a cada etapa y grupo temático; elaboración, para cada fase histórica, de modelos tecnológicos en que se integraban las necesidades sociales en torno a la malaria con los conocimientos epidemiológicos sobre la enfermedad y las prácticas de intervención. Al presentarse cada modelo se ha procurado destacar sus características más importantes.

El universo de los documentos que proporcionaron las bases empíricas de la investigación abarcó, entre otras cosas, los informes de la Inspectoría de la Profilaxis del Paludismo (19231932), el Servicio de Profilaxis de la Malaria (1933-1958), el Servicio de Erradicación de la Malaria y de la Profilaxis de la Enfermedad de Chagas (1959-1969), la Superintendencia de Saneamiento Ambiental (1970-1974) y la Superintendencia del Control de las Enfermedades Endémicas (19751994); separatas de artículos, manuales de adiestramiento, registros de servicios, materiales propagandísticos, informes de reuniones nacionales e internacionales, programas de trabajo, y colecciones y archivos de la Biblioteca Geraldo Buralli de la Superintendencia del Control de las Enfermedades Endémicas (SUCEN) para el período de 1900 a 1994; la Revista Brasileira de Malariologia e Doenças Tropicais (1949-1986); la Revista da Fundação Serviços Especiais de Saúde Pública (19471985); informes del Comité de Expertos en Paludismo de la OMS (1947-1986); informes del Servicio Nacional de Malaria y de las Reuniones de Directores de Servicios Nacionales para la Erradicación de la Malaria archivados en la biblioteca de la SUCEN; dos infor- mes, uno de ellos titulado Estado de la erradicación de la malaria en las Américas, publicado por la OPS de 1962 en adelante, y el otro Case studies in epidemiology of malaria, publicado por la OMS a partir de 1971; la obra Quarterly Bibliography of Major Tropical Diseases, publicada por la OMS a partir de 1980; libros de texto sobre la malaria por autores conocidos, editados en el siglo $\mathrm{XX}$ y pertenecientes a la Biblioteca de la SUCEN y a la biblioteca personal donada por el doctor Firmino de Oliveira al Departamento de Medicina Social de la Santa Casa de São Paulo.

\section{MODELO ECOLÓGICO AMBIENTAL, 1930 A 1950}

En el estado de São Paulo, el período de 1930 a 1950 correspondió a la transición de una economía predominantemente agraria y exportadora a una economía industrial. Fue una época de transformaciones en todo el estado. En las zonas que habían sido habitadas desde más temprano (1532 a 1886) se acentuó el parcelamiento de las grandes propiedades rurales. El cultivo de una variedad de productos para consumo interno reemplazó a la producción con fines de exportación y se impulsó la creación de industrias. En las zonas que fueron ocupadas de 1880 a 1920, se diversificó la producción agrícola y hubo una gran inmigración de otras partes del Brasil y del extranjero. Por último, la zona cuya colonización fue más tardía seguía teniendo una producción con fines de exportación, principalmente de café y algodón, y los índices de migración eran los más altos de todo el estado.

La población del estado de São Paulo era predominantemente rural y la mayor parte de la población económicamente activa se dedicaba a actividades agrícolas. Existía, por lo tanto, un conjunto de factores que favorecían la aparición de la malaria: población rural, ocupación y edificación de áreas nuevas, grandes corrientes migratorias y construcción de obras de infraestructura, tales como centrales hidroeléctricas y carreteras $(1,2)$.
Para el trabajador rural tener malaria implicaba una grave limitación de su capacidad vital, es decir, la imposibilidad de realizar adecuadamente sus actividades cotidianas. Para la sociedad, la malaria significaba una capacidad laboral reducida y una consiguiente pérdida de productividad y del volumen de producción. La infección de un número relativamente grande de trabajadores del área rural tenía, por consiguiente, grandes consecuencias. La malaria surgió como obstáculo al proceso de desarrollo y exigió la creación de instituciones destinadas a combatirla. Durante ese período las tasas de incidencia fueron altas $y$ puede que hayan llegado a ser mayores de 200 casos por 100000 habitantes, salvo en los primeros años, cuando había pocos puestos de diagnóstico en el estado (cuadro 1).

La distribución de casos en el territorio aún reflejaba las diversas etapas de la colonización y demostraba que la aparición de la enfermedad era el resultado, básicamente, de las condiciones de vida. En las zonas más antiguas la incidencia era baja o nula, excepto en la zona del litoral, donde las tasas eran más altas debido a la gran densidad del vector. El uso de las tierras para fines agrícolas o de urbanización, la estabilidad de las poblaciones, y el crecimiento urbano e industrial habían creado condiciones desfavorables para la aparición de la enfermedad, y en tales áreas había un

\section{CUADRO 1. Tasas de incidencia de malaria (por 100000 habitantes) en el estado de São Paulo, Brasil, 1933 a $1950^{a}$}

\begin{tabular}{rrrrrr}
\hline Año & Tasa & Año & Tasa & Año & Tasa \\
\hline 1933 & 52 & 1939 & 337 & 1945 & 690 \\
1934 & 79 & 1940 & 714 & 1946 & 318 \\
1935 & 477 & 1941 & 2423 & 1947 & 282 \\
1936 & 143 & 1942 & 285 & 1948 & 88 \\
1937 & 212 & 1943 & 268 & 1949 & 86 \\
1938 & 224 & 1944 & 236 & 1950 & 261 \\
\hline
\end{tabular}

Fuente: Datos de población del Instituto Brasileño de Geografía y Estadística.

a Los datos correspondientes al período de 1933 a 1938 fueron proporcionados por la Inspectoría de la Profilaxis del Paludismo, y los correspondientes al período de 1939 a 1950 por el Servicio de Profilaxis de la Malaria. 
predominio de casos esporádicos. En la zona de transición donde predominaban las propiedades pequeñas o las fincas con pocos trabajadores, la incidencia también solía ser baja. Las tasas eran altas en la zona de transición y en las áreas nuevas, sobre todo en aquellos lugares donde las propiedades eran grandes y donde había un sistema de posesión de tierras que atraía a un gran número de inmigrantes. Asimismo, las epidemias eran más frecuentes en las zonas recién ocupadas debido a la tala de bosques y a los continuos movimientos migratorios (3).

En lo referente a la malaria, prevalecía el concepto de salud-enfermedad que se podría llamar ecológicoambiental, dado el papel central que se otorgaba al medio ambiente. Predominaba la idea de que la malaria se producía como consecuencia de la interacción de tres grupos de factores - unos de tipo etiológico, otros relacionados con el vector y otros vinculados con el huésped-, todos mediados por el ambiente. Este último comprendía una serie de variables y circunstancias que eran necesarias para la supervivencia de las poblaciones de plasmodios, anofelinos y seres humanos y que favorecían el contacto entre ellas, y eso permitía que se consolidara el llamado "mecanismo de reservorio", es decir, que se instalara el ciclo de transmisión. El ambiente ocupaba en el modelo el lugar de la estructura social y permitía la "naturalización" del proceso de salud-enfermedad. Las diversas variables se estudiaban como un conjunto no estructurado de factores relacionados de manera lineal, pareada o secuencial. De ahí el énfasis que se ponía en la designación ecológicoambiental.

Los estudios entomológicos se intensificaron y se establecieron criterios para evaluar la capacidad vectorial de cada especie. Se observó que la distribución de la enfermedad se vinculaba cada vez más con la distribución de los vectores. En el estado de São Paulo, los vectores principales de la enfermedad eran Anopheles $(N)$ darlingi en el altiplano; $A$. (N) tarsimaculatus, $A$. (K) cruzi y $A$. (K) bellator en el litoral; $A$. (N) stro- dei específicamente en el municipio de Marilia y $A$. (N) albitarsis exclusivamente en las poblaciones costeras de Iguape y Cananéia (4-8). ${ }^{3}$

Las prácticas de intervención durante ese período se limitaban al control de las larvas mediante pequeñas obras de ingeniería orientadas a reducir el número de criaderos de cualquier especie de anofelinos. A pesar de que estas medidas se dirigían a modificar el ambiente, su efecto fue relativamente pequeño, ya que la escasez de recursos financieros impidió que las obras se extendieran hasta el punto de modificar de manera importante la densidad vectorial. A menudo las autoridades inspectoras recorrían las prefecturas y visitaban a los propietarios de las tierras para que financiaran y ejecutaran esas obras y se limitaban a darles orientación técnica.

Al ampliarse los conocimientos al respecto, la labor de combatir los vectores se centró en la llamada "vigilancia de focos", que consistía en identificar y contar los criaderos, estudiar e identificar las larvas que desempeñaban una función en la transmisión, hacer tratamientos con petróleo o con solución verde de París, y hacer un seguimiento. La necesidad de observar esta rutina y la gran extensión de los depósitos de agua en el estado, sumadas a las dificultades de identificar los criaderos de $A$. (N) darlingi, limitaban mucho la aplicación de esta estrategia (9). ${ }^{4}$

La existencia de conocimientos técnicos y de estrategias de intervención no garantizaba que las mismas se pusieran en práctica, puesto que no lo permitían las peculiaridades de la organización social en São Paulo. La misma organización social que fomentaba la expresión insuficiente de las necesidades de salud y que las reducía a restricciones aplicadas a la fuerza de trabajo, también limitaba la aplicación del saber técnico, cuya distribución era

\footnotetext{
3 Correa RR, Lima FO. Antropofilia do $A$. (N) albitarsis domesticus, $A$. (N) darlingi e Culex fatigans; São Paulo: Servicio de Profilaxis de la Malaria; 1990. (Documento mimeografiado).

4 Unti O. SPM: sua atividade no passado, presente e no futuro; São Paulo: Servicio de Profilaxis de la Malaria; 1951. (Documento mimeografiado).
}

inevitablemente desigual. El propósito principal del control de la malaria en São Paulo durante ese período era mantener a la fuerza de trabajo empleada en el sector agrario y en la construcción de obras infraestructurales. Dadas las limitaciones financieras ya señaladas, estas últimas y las empresas agroindustriales, junto con las zonas urbanas de gran actividad económica, se consideraban de importancia prioritaria para el control de la transmisión.

En el período de 1930 a 1950 la malaria era común en prácticamente todo el estado. Se observaban tasas de incidencia altas y epidemias cada 5 años, y la transmisión era predominantemente rural. Prevalecía el concepto ecológico-ambiental, es decir, la creencia en que numerosos factores sin jerarquización, fragmentados y correlacionados de forma lineal, determinaban la aparición de la enfermedad. Entre ellos tenían mayor relieve los que se vinculaban con el ambiente físico, en tanto afectaban directamente a la supervivencia y proliferación de los vectores. La encuesta epidemiológica surgió como instrumento principal para el diagnóstico de la situación y el control se basó en la noción de malaria como problema local, es decir, como enfermedad de alcance colectivo que se expresaba de modo particular en función de las características locales. El control se centró en reducir las poblaciones de vectores por medio de la eliminación o tratamiento de criaderos, con miras a disminuir la densidad de los mosquitos.

La estrategia de intervención, en el entretiempo, tenía que ajustarse a las necesidades de las actividades de control, que debían extenderse a toda la población y adaptarse a las características socioeconómicas de la sociedad de São Paulo, y para lograrlo se establecieron prioridades. A pesar de las iniciativas destinadas a organizar y extender las actividades de control, a fines de los años cuarenta la malaria representaba un problema de salud pública importante en el estado de São Paulo.

Cuando se acabó la Segunda Guerra Mundial, la OMS creó el Comité de 
Expertos en Paludismo, que en 1947 emitió las principales pautas para el control de la enfermedad. El primer informe, que data de 1947, destacaba los adelantos técnicos y ponía énfasis en la síntesis de medicamentos nuevos, como la cloroquina, y en el uso de larvicidas de acción residual. El segundo informe, que apareció en 1948, proponía la creación de organismos nacionales para el control "e incluso la erradicación" de la malaria. Con ello se inició una nueva fase en las actividades de control en el ámbito mundial $(10,11)$.

\section{MODELO TÉCNICO BASADO EN LA APLICACIÓN DE CAMPAÑAS, 1950 A 1970}

A principios de los años cincuenta, quedaba apenas $18 \%$ de la cubierta vegetal original del estado de São Paulo. Dicho estado era el principal productor de nueve de los productos agrícolas más importantes del país, aunque ya en 1950 su población urbana era más numerosa que su población rural. La transformación del perfil urbano-rural de la población de São Paulo fue el resultado de dos movimientos independientes: un aumento de la empleomanía en el sector industrial, favorecido por el influjo de capitales extranjeros después de las modificaciones de la ley de remisión de ganancias; y la intensificación del proceso de mecanización agrícola, acompañada de la disolución del sistema de colonización y de la transformación de los colonos en trabajadores itinerantes o "flotantes" que abandonaban el campo y se instalaban en la periferia de los núcleos urbanos $(1,12$, 13). En la década de 1960 esas transformaciones se intensificaron, con el resultado de que se produjo una reducción absoluta y relativa de la población rural, incluso en aquellos lugares donde el trabajo agrícola seguía predominando. Al final de ese período la población urbana llegó a representar $80 \%$ de la población total y se observaba una concentración demográfica marcada en los polos industriales del estado.
Las nuevas condiciones de la organización económica y social también alteraron la frecuencia de la malaria. Después de la fase inicial de deforestación y colonización del territorio, en que la incidencia de la enfermedad solía ser alta, en la fase de consolidación de la ocupación agrícola la incidencia tendió a reducirse. Esta tendencia también se vio reforzada por la reducción del número de trabajadores rurales y por las modificaciones ambientales producidas por la industrialización y urbanización, como la contaminación de los ríos por desperdicios y emanaciones industriales y por las redes de aguas negras, que alteraron los criaderos de A. $(\mathrm{N})$ darlingi $(14,15)$.

Durante los años cincuenta, las tasas de incidencia eran de alrededor de 30 casos por 100000 habitantes y se redujeron notablemente en los años sesenta debido a la campaña de erradicación. Había exacerbaciones epidémicas cada 5 años. Aún persistía la distribución desigual dentro del territorio, ya que la concentración de casos autóctonos y las tasas de incidencia eran mayores en la zona nueva, principalmente en la "frontera" con el estado de Matto Grosso (cuadro 2).

En el plano económico, la existencia del trabajador rural con malaria adquirió un nuevo significado. Para el trabajador itinerante la enfermedad se traducía en menos días de trabajo y, por consiguiente, en menos paga, mientras que para el "productor rural" la endemia ya no tenía la misma importancia. Las necesidades de salud de los trabajadores podían expresarse en términos colectivos y su definición provenía del Estado, como parte de una política de desarrollo que suponía la modernización de la producción agrícola. El trabajador representaba el peligro de que se introdujera la transmisión en las áreas urbanas, con lo que ponía en riesgo a los trabajadores industriales. Este peligro dio ímpetu a los compromisos ya asumidos por el gobierno federal y los organismos internacionales, como la OPS, en cuanto a la adopción de un programa de erradicación.

La introducción de la cloroquina y del plaguicida diclorodifeniltricloroetano (DDT) como vehículos de control a partir de 1947 significó, en la práctica, que la malaria se consideraba una enfermedad de las masas y ello permitió que la intervención se ampliara de formas previamente inconcebibles. La facilidad con que se administraba la cloroquina y la eficacia obtenida con un solo tratamiento con cloroquina permitieron su distribución a gran escala y por personas sin adiestramiento. Los rociamientos intradomiciliarios con DDT de efecto residual prolongado fueron una manera más sencilla y relativamente barata de combatir los vectores. En su conjunto, estos nuevos instrumentos dieron a los malariólogos la esperanza de poder

\section{CUADRO 2. Tasas de incidencia total de malaria y de casos autóctonos (por 100000 habi- tantes) en el estado de São Paulo, 1951 a $1970^{\text {a }}$}

\begin{tabular}{lccccc}
\hline Año & $\begin{array}{c}\text { Tasa } \\
\text { total }\end{array}$ & $\begin{array}{c}\text { Tasa de casos } \\
\text { autóctonos }\end{array}$ & Año & $\begin{array}{c}\text { Tasa } \\
\text { total }\end{array}$ & $\begin{array}{c}\text { Tasa de casos } \\
\text { autóctonos }\end{array}$ \\
\hline 1951 & 80 & 17 & 1961 & 59 & 37 \\
1952 & 45 & 8 & 1962 & 28 & 12 \\
1953 & 29 & 8 & 1963 & 16 & 1 \\
1954 & 51 & 16 & 1964 & 13 & 1 \\
1955 & 49 & 17 & 1965 & 10 & 2 \\
1956 & 29 & 10 & 1966 & 11 & 4 \\
1957 & 37 & 14 & 1967 & 8 & 2 \\
1958 & 32 & 28 & 1968 & 6 & 1 \\
1959 & 31 & $\ldots$ & 1969 & 5 & 1 \\
1960 & 87 & 59 & 1970 & 4 & 0,2 \\
\hline
\end{tabular}

Fuente: Datos de población del Instituto Brasileño de Geografía y Estadística.

a Los casos correspondientes al período de 1951 a 1958 se obtuvieron del Servicio de Profilaxis de la Malaria, y los del período de 1959 a 1970 de los Servicios de Erradicación de la Malaria y de la Profilaxis de la Enfermedad de Chagas. 
reducir notablemente la transmisión de la malaria, o incluso de interrumpirla, en las zonas endémicas.

La controversia inicial sobre si la conducta más adecuada era erradicar el vector o la enfermedad se empezó a resolver después del fracaso de la tentativa de erradicación de $A$. labranchiae en Sardeña. Aunque no se logró erradicar el vector de la isla, al menos se demostró la factibilidad de interrumpir la transmisión y, por consiguiente, de erradicar la enfermedad (16). La insistencia de los organismos internacionales en la erradicación de la enfermedad no surtió efectos inmediatos en los países, pues estaba en pugna con la actitud escéptica de muchos malariólogos, y las reducciones importantes de la incidencia logradas mediante el uso de DDT en los programas de control tornaron innecesario el esfuerzo exigido por las actividades de erradicación. La aparición de resistencia a los insecticidas residuales entre los anofelinos, que fue observada en varios países, alteró por completo ese panorama. La resistencia significaba el riesgo de perder el mejor instrumento de control creado hasta ese momento y ponía en peligro los avances ya alcanzados y la continuación de las actividades.

La campaña, estrategia escogida frente al riesgo inminente de una resistencia generalizada, se concibió como una operación en cuatro fases. En la fase preparatoria, que duró un año, se hizo una encuesta preliminar para poder delimitar la zona con malaria y planificar y organizar las medidas. La fase de ataque, cuya duración fue de 2 o 3 años, consistió en rociar con DDT todos los domicilios en la zona rural, en ciclos periódicos, hasta interrumpir la transmisión. La fase de consolidación se basó en la búsqueda activa y en el tratamiento radical de todos los casos sospechosos y en la eliminación de focos de transmisión residuales. Por último, la fase de mantenimiento se caracterizó por la puesta en marcha del sistema de vigilancia epidemiológica con el fin de prevenir la reactivación de la transmisión (17).

El concepto ecológico-ambiental fue abandonado y en su lugar una serie de "principios técnicos" pasaron a orien- tar las acciones. La doctrina de la erradicación se apoyaba en tres dogmas: la autolimitación de la infección, su transmisión exclusivamente intradomiciliaria y la posibilidad de interrumpirla mediante la reducción de los mosquitos adultos infectados. Estas condiciones, siendo las mismas en todas partes del mundo salvo en el África, permitían la aplicación de una estrategia común. Ya no tenía cabida la noción de una enfermedad compleja cuyos factores determinantes variaban de un lugar a otro y que se expresaba de manera particular en distintas circunstancias. La malaria en ese momento se concebía en términos de laminillas positivas y vectores infectados.

En São Paulo, la campaña empezó en 1959 en 198 municipios - casi 40\% de la superficie territorial del estadoque constituían la zona malárica y que tenían cerca de 2,7 millones de habitantes ( $23 \%$ de la población estatal). La fase de ataque se puso en práctica de 1960 a 1963 con el rociamiento de $98 \%$ de los domicilios en la zona de malaria en cada ciclo semestral. El rociamiento diario estaba cuidadosamente programado, regularizado y supervisado. $\mathrm{La}$ búsqueda activa de personas con fiebre redundaba en el sometimiento anual de cerca de $10 \%$ de la población al examen parasitológico con microscopio. Se crearon 5000 puestos de notificación para la búsqueda pasiva y el tratamiento de casos sospechados. Las palabras clave eran "oportunidad", "adecuación" y "regularidad". Cada acción debía adecuarse a la disponibilidad de recursos y ejecutarse de forma oportuna, con garantía de su continuidad y de un avance progresivo hacia la meta establecida.

En 1968, al completarse la primera evaluación oficial del programa, la zona libre de malaria contenía $68 \%$ de la población; el área en consolidación abarcaba $31 \%$ de la población y 53\% del territorio, y la zona de ataque se encontraba reducida a $3 \%$ del territorio (16 municipios con $1 \%$ de la población).

La campaña de erradicación, como estrategia de acción, aprovechaba los instrumentos técnicos disponibles $\mathrm{y}$ desarrollaba intervenciones de la mayor eficacia posible. La estandarización de las actividades permitió y facilitó su extensión a todos los lugares, con el uso de relativamente pocos profesionales especializados. La estructura muy jerarquizada, en que las tareas de cada trabajador estaban perfectamente definidas y explicadas en todo detalle en los manuales de instrucción, así como el sistema permanente y minucioso de registros y la supervisión continua, permitieron usar los recursos humanos, materiales y fondos de modo racional, aprovechándolos al máximo y potenciando sus efectos.

La estrategia basada en campañas, con su aparato normativo, representaba la posibilidad de extender a todos, indiscriminadamente, las actividades de control con el fin de modificar radicalmente las condiciones en que se producía la enfermedad por medio de una intervención técnica planificada y ejecutada con rigor, relativamente corta y extendida a todo el territorio. Con ello se superaban, con mucho, las limitaciones del modelo anterior.

Una vez iniciada la campaña, la reducción de la incidencia fue continua. Durante la fase de ataque los casos sufrieron una reducción de $78 \%$ y los de tipo autóctono prácticamente desaparecieron (con una reducción de 98\%). El índice de laminillas positivas bajó a 0,2\%, es decir, se redujo 19 veces, y el índice parasitario anual se redujo a 0,03 laminillas positivas por 1000 habitantes. El resultado de la campaña fue muy bueno, puesto que la interrupción total tardó 4 años en lograrse, como se había anticipado.

Al compararse la incidencia en el período de 1947 a 1958, que precedió a la campaña, con la incidencia posterior al inicio de la misma, se advierte que con la estrategia a base de campañas se produjo una organización tecnológica del trabajo que, de hecho, tuvo un impacto en la frecuencia de la enfermedad. Prácticamente los mismos instrumentos técnicos de intervención fueron utilizados en ambos momentos: los rociamientos con DDT y los tratamientos con cloroquina. Pero no fue hasta el segundo período, cuando transcurría la campaña, que las accio- 
CUADRO 3. Tasas de incidencia total de malaria y de casos autóctonos (por 100000 habitantes) en el estado de São Paulo, 1971 a 1990a

\begin{tabular}{cccccc}
\hline Año & $\begin{array}{c}\text { Tasa } \\
\text { total }\end{array}$ & $\begin{array}{c}\text { Tasa de casos } \\
\text { autóctonos }\end{array}$ & Año & $\begin{array}{c}\text { Tasa } \\
\text { total }\end{array}$ & $\begin{array}{c}\text { Tasa de casos } \\
\text { autóctonos }\end{array}$ \\
\hline 1971 & 5 & 0,2 & 1981 & 5 & 0,1 \\
1972 & 5 & 0,4 & 1982 & 5 & 0,1 \\
1973 & 4 & 0,1 & 1983 & 6 & 0,1 \\
1974 & 5 & 0,3 & 1984 & 7 & 0,2 \\
1975 & 6 & 0,2 & 1985 & 8 & 0,3 \\
1976 & 5 & 0,1 & 1986 & 9 & 0,2 \\
1977 & 6 & 0,7 & 1987 & 10 & 0,1 \\
1978 & 5 & 0,1 & 1988 & 8 & 0,1 \\
1979 & 5 & 0,2 & 1989 & 8 & 0,1 \\
1980 & 5 & 0,2 & 1990 & 6 & 0,1 \\
\hline
\end{tabular}

Fuente: Datos de población del Instituto Brasileño de Geografía y Estadística.

a Los casos correspondientes al período de 1971 a 1974 se obtuvieron de la Superintendencia de Saneamiento Ambiental, y los del período de 1975 a 1990 de la Superintendencia del Control de las Enfermedades Endémicas.

nes de control se extendieron lo suficiente para modificar radicalmente la presencia de la malaria en São Paulo.

\section{MODELO FOCAL Y PREVENTIVO, 1970 A 1990}

Las transformaciones socioeconómicas y demográficas que se fueron generando en el estado de São Paulo a partir de la década de 1950, sumadas al impacto producido por la campaña de erradicación, modificaron por completo la epidemiología de la malaria. La mecanización de la agricultura fue aun más acentuada que en el período anterior y el trabajo temporero adquirió carácter predominante, debido a la inmigración de trabajadores de estados vecinos, especialmente durante las cosechas. La población rural, que en 1970 representaba $11,4 \%$ de la población total, bajó a escasamente $5 \%$ en 1991 (1).

En ese período los casos de malaria autóctonos fueron pocos, incluso menos de 100 al año, y muchos aparecieron a raíz de la importación de casos cuya transmisión había ocurrido fuera del estado. La malaria dejó de ser un problema perenne para la población de São Paulo y se convirtió en un problema latente cuya aparición dependía de la situación epidemiológica en otras partes del país (cuadro 3 ).
A pesar de que la campaña de erradicación había planteado algunas dificultades, cuando se aplicó en el resto del país, su impacto también fue notable: en la fase que le precedió los casos nuevos se estimaban en cerca de 5 millones al año, pero en 1974, cuando todas las áreas habían pasado por la fase de ataque, solo se registraron 50000 casos. Simultáneamente, la ocupación del territorio amazónico en el centro de la zona oriental provocó un aumento notable de la transmisión durante la segunda mitad de la década de los setenta y en toda la década siguiente $(18,19) .{ }^{5}$ El efecto de los cambios que estaban sucediendo en la zona amazónica se haría sentir en todas las áreas donde se había interrumpido la transmisión de la enfermedad, incluido el estado de São Paulo. El flujo constante y creciente de personas entre la zona endémica y las áreas libres de transmisión creó la necesidad de mantener sistemas de vigilancia epidemiológica que permitieran identificar y controlar oportunamente los brotes epidémicos provocados por la penetración de portadores (fuentes de infección) en las áreas

\footnotetext{
Marques AC, Pinheiro EA, Motta EGF. Fatores que dificultam o controle da malária na região amazônica. São Paulo: Superintendencia del Control de las Enfermedades Endémicas; 1977. (Documento mimeografiado).
}

con vectores y una población susceptible.

En los años setenta, la tasas de incidencia de malaria en São Paulo se mantuvieron en alrededor de 5 casos por 100000 habitantes, aumentaron progresivamente de 1983 a 1987, y posteriormente volvieron a bajar. De los casos registrados en 1983, 98\% fueron "importados" del área endémica, especialmente de Rondônia (Pôrto Velho y Ariquemes), Mato Grosso (Colíder y Alta Floresta) y Pará (Itaituba, Marabá y Concepción de Araguaia). Los individuos afectados fueron los campesinos, mineros y camioneros que se desplazaban a menudo de un lado a otro entre el área endémica y el estado de São Paulo. La epidemiología de la enfermedad durante ese período se caracterizó por la persistencia de la transmisión en las áreas maláricas del litoral, reintroducciones esporádicas de casos en el altiplano y un número creciente de casos importados. Después de 1987 los casos en usuarios de drogas inyectadas empezaron a aumentar $(20,21)$.

Al final de ese período, la malaria era un problema de carácter focal, tanto en el Brasil en general como en el estado de São Paulo en particular. En la zona amazónica, relativamente pocos municipios eran los responsables de casi todos los casos de malaria en el país. En 1990, prácticamente 85\% de todos los casos provenían de apenas 79 municipios. São Paulo y Paraná eran los estados más vulnerables, pues a ellos llegaban y en ellos se diagnosticaban la mayor parte de los casos que venían del exterior, desde la zona amazónica. En São Paulo, 16 municipios tenían en aquel momento transmisión natural activa, mientras que 224 municipios, esparcidos por todo el territorio, registraban casos importados que ponían de manifiesto la diseminación de las fuentes de infección.

En aquel período urgía mantener interrumpida la transmisión, reducir los focos residuales y controlar a tiempo cualquier foco nuevo. El trabajo de control pasó a ser dirigido por cada localidad, y no por las municipalidades. Las actividades, basadas fun- 
damentalmente en la vigilancia epidemiológica, se dirigieron a los grupos y territorios de mayor vulnerabilidad.

Aunque mantener libre de la enfermedad a la población del estado parecía más fácil en las condiciones que imperaban en aquel momento, dos dificultades saltaban a la vista: por un lado, la presencia continua de casos importados imponía la necesidad de una vigilancia ágil y oportuna; por otro, las autoridades se mostraban poco interesadas en proveer los recursos necesarios para controlar un problema que era una mera amenaza y no una realidad. La estructura del programa en el estado había dado muestras de eficiencia, puesto que había impedido que se reintrodujera la transmisión, controlado los focos residuales y evitado la creación de focos nuevos. Por añadidura, había permitido investigar y controlar situaciones imprevistas y más difíciles de manejar, como la aparición de casos en usuarios de drogas inyectadas. Poco a poco la enfermedad dejó de constituir un peligro para la población en general y empezó a afectar principalmente a ciertos grupos de individuos en función de su trabajo, si exigía un desplazamiento a zonas endémicas, o de sus hábitos personales, como el uso de drogas y la exposición durante actividades de recreo al aire libre.

Según indica la experiencia adquirida, una vez que se ha logrado interrumpir la transmisión, la endemia difícilmente se reinstala en áreas con un buen desarrollo socioeconómico. El temor a que la persistencia de la transmisión en zonas vecinas menoscabe las iniciativas de control no está respaldado por los hechos observados en las últimas décadas.

\section{REVISIÓN DE LA CAMPAÑA DE ERRADICACIÓN EN LOS AÑOS NOVENTA}

Desde mediados de los años setenta, el deterioro de la situación epidemiológica en los países subdesarrollados mermó la creencia en la posibilidad de erradicar la malaria en el corto plazo y llevó a la OMS a recomendar una revisión de la estrategia fundada en la ejecución de campañas. Al principio, el llamado "resurgimiento" de la malaria se consideró el resultado de deficiencias técnicas y logísticas en la ejecución de estas últimas, sumadas a una reducción de los recursos financieros y a cierto debilitamiento de la decisión política de erradicar la enfermedad. Posteriormente se atribuyó a la aplicación poco consciente de la estrategia fundada en la ejecución de una campaña, sin tener conocimiento alguno de los diferentes perfiles epidemiológicos locales (22-24).

La creencia de que la malaria ha recrudecido como resultado de una estrategia mal ejecutada llevó a un esfuerzo por subsanar las deficiencias de la campaña de erradicación, sin modificar la propuesta en sus rasgos esenciales. Poco a poco, el persistente aumento de la incidencia alteró las nociones que se tenían en torno a la enfermedad y de ahí nació la "teoría focal". El concepto de foco surgió cuando se hizo evidente que la enfermedad tenía manifestaciones muy heterogéneas en diferentes contextos epidemiológicos. Se trató de encontrar los motivos por los cuales en algunos focos no se había podido interrumpir la transmisión. En vez de colocar el énfasis en el "fracaso" de la erradicación, se asumió una actitud afirmativa centrada en la noción de foco. Las consecuencias inmediatas de este nuevo enfoque fueron la recuperación del antiguo concepto de estratificación epidemiológica, la diversificación de las técnicas y actividades de control y el reconocimiento del valor de la atención primaria como modelo de organización para la atención de casos clínicos.

El concepto de foco no permitía reducir a términos demasiado simplistas el binomio representado por el vector y la laminilla positiva y se apoyaba en relaciones arraigadas en el concepto de estratificación epidemiológica. Esta última se propuso como instrumento para identificar zonas con un riesgo homogéneo y orientar la aplicación de "tecnologías apropiadas, científicamente adecuadas y aceptables y ase- quibles en el sentido social" (25-27). El modelo fue objeto de críticas conceptuales y operativas. En el plano teórico, la objeción principal fue la prioridad otorgada a categorías de tipo espacial, puesto que la estratificación era territorial, sin reparo en los cambios producidos a lo largo del tiempo. Las deficiencias operativas se debían a las limitaciones propias de las bases de datos creadas según divisiones geopolíticas donde raras veces existían situaciones epidemiológicas homogéneas en lo referente a la malaria, y a la necesidad de realizar encuestas largas y costosas para obtener información cuando no se disponía de registros (25). ${ }^{6}$

Ante estas críticas, se propuso abandonar los estratos de riesgo y adoptar paradigmas o modelos en que los factores determinantes de carácter ecológico y social se expresaran en términos de "tipos ideales" de utilidad para elaborar patrones de control adaptados a distintas situaciones epidemiológicas $(28,29)$. El énfasis en las carácterísticas locales fue aun más acentuado en los enfoques "microepidemiológicos" que surgieron a fines de los años ochenta y que se apoyaban en el estudio antropológico de individuos y grupos familiares, con lo que la unidad de análisis se redujo a los individuos expuestos al riesgo (30).

El concepto de erradicación fue abandonado y desplazado paulatinamente por el enfoque de riesgo, que en un inicio se aplicó a los diversos estratos de la población, posteriormente a situaciones epidemiológicas paradigmáticas, y por último a la unidad familiar y a los individuos. En el transcurso de este proceso, lo general fue reemplazado por lo particular y las prácticas de la salud pública, de alcance poblacional, cedieron el paso a las prácticas preventivas para la protección del individuo.

Las orientaciones internacionales relacionadas con el control de la enfer-

\footnotetext{
6 Rubio-Palys Y. Malaria in the Americas: ecological/epidemiological paradigms; São Paulo: Superintendencia del Control de las Enfermedades Endémicas; 1992. (Documento mimeografiado).
} 
medad se alteraron radicalmente durante la Conferencia Ministerial promovida por la OMS en 1992 en Amsterdam, Países Bajos (31). De acuerdo con las directrices aprobadas por los ministros, el control de la malaria se debía basar en tres grupos de actividades. El primero, denominado "manejo de la enfermedad", incluía todo lo relacionado con el diagnóstico y tratamiento de los casos. Los gobiernos eran los encargados de garantizar los medios para que los individuos enfermos recibieran el diagnóstico correcto y el tratamiento adecuado. No se buscaba eliminar las fuentes de infección, sino reducir la morbilidad y mortalidad por malaria. Las actividades de búsqueda activa de casos debían suspenderse y el "manejo de la enfermedad" delegarse a los servicios de atención primaria. El segundo grupo de actividades, dedicado a la "prevención de la enfermedad", incluía las medidas para la protección individual, la quimioprofilaxis, la inmunización y el control domiciliario de los vectores. El enfoque de las actividades de control se desplazó del medio ambiente y de la protección de los posibles huéspedes hacia los individuos y sus "conductas riesgosas". El tercer grupo de actividades, destinado a "la prevención y control de epidemias", abarcaba las actividades tradicionales de vigilancia epidemiológica y seguía incluyendo al mismo tiempo intervenciones colectivas de cuya eje- cución el estado seguía siendo el principal responsable.

Al transferirse a los individuos la responsabilidad de su propia protección y dejarse a los programas la tarea de garantizar o proveer los recursos necesarios para el diagnóstico y tratamiento de casos, surgía la posibilidad de que se empeorara la falta de equidad (32). Las actividades colectivas se aplicaban a todos los individuos, fueran o no susceptibles a contraer la enfermedad, independientemente de su conducta individual; estaban más orientadas hacia la sociedad en su totalidad que hacia cada persona en lo particular. Las actividades de control con enfoque individual afectaban, a su vez, a cada una de las personas que las practicaban; las condiciones en que se producía la enfermedad ahora quedaban sujetas al comportamiento de cada individuo. De este modo la falta de equidad propia de la organización social tendía a reproducirse en la estructura del control, ya que a las personas en los estratos marginados de la sociedad, quienes solían tener el mayor riesgo de enfermar, también les resultaba más difícil adoptar las medidas de protección recomendadas o acudir a los establecimientos de atención.

Con la nueva estrategia de control prácticamente se abandonaba la meta de poner al alcance de todo el mundo los avances de la ciencia y de la técnica, según los ideales de "igualdad y fraternidad". Se daba por sentado que existían desigualdades socioeconómicas y que no eran remediables por medio de intervenciones técnicas. La falta de control de la malaria en los países en desarrollo no representaba una amenaza para los países desarrollados y por lo tanto dejaba de ser necesaria una iniciativa mundial para hacer frente al problema. Cada país debía tratar de sopesar sus necesidades a la luz de sus propias posibilidades de acción, valiéndose de productos técnicos y científicos que facilitaran su desarrollo económico. La máxima humanista de "a cada cual según sus necesidades" quedó reemplazada por la regla liberal de "a cada cual según sus posibilidades" (es decir, económicas y políticas).

El pragmatismo y la rendición frente a las dificultades que entraña extender el control a las zonas donde imperan condiciones muy desfavorables para el desarrollo socioeconómico, fenómenos que formaban parte de la estrategia actual, coincidían perfectamente con "el nuevo orden mundial" en que los "ineptos" que no estaban en capacidad de acumular capital para su propio beneficio se iban quedando al margen del sistema, creando desigualdades sociales profundas y problemas irresolubles. En ese nuevo orden de cosas, solo los individuos con una visión "romántica" y "utópica" de las cosas esperarán con idealismo poético un futuro en que todos los seres humanos puedan convivir en completa armonía.

\section{REFERENCIAS}

1. Fundaçao SEADE/USP. Atlas de população do Estado de São Paulo. São Paulo: Fundação SEADE/USP; 1992.

2. Monbeig P. Pioneiros e fazendeiros de São Paulo. São Paulo: Editora HUCITEC/Polis; 1984.

3. Costa Filho A. Relatório do periodo 1933-1937. São Paulo: Inspetoria de Prophilaxia do Impaludismo; 1937.

4. Ayrosa Galvão AL, Lane J. Notas sobre os Nyssorhincus de São Paulo. Rev Biol Hig 1937; 9:37-45.

5. Correa RR. Da infecção natural pela plasmodiose malárica do A. (K) cruzi. Fol Clin Biol 1943;XV:23-32.

6. Correa RR, Ramos AS, Rachou R. Anofelismo e malária em algumas represas em São Paulo. Fol Clin Biol 1943;XV:33-43.
7. Correa RR, Ramos AS. Os anofelinos da ilha de Santo Amaro. Arq Hig Saude Publica 1944; IX:7-16.

8. Correa RR, Lima FO, Ramos AS. Os anofelinos do subgênero kertzsia: sua relação com a malária no Estado de São Paulo. Dusenia 1950;1:203-214.

9. Russel P. Malaria and society. J Nat Malaria Soc 1951;10:1-7.

10. Organisation mondiale de la Santé. Comité d'experts sur le paludisme: rapport sur la première session. Bull World Health Organ 1947; 1:23-32.

11. Organisation mondiale de la Santé. Comité d'experts sur le paludisme: rapport sur la deuxième session. Bull World Health Organ 1948;1:235-279.
12. Cano W. Desequilíbrios regionais e concentração industrial no Brasil 1930-1970. Campinas: Editora Global; 1985.

13. Silva LJ. Evolução da doença de Chagas no Estado de São Paulo [tesis de doctorado] Faculdade de Medicina de Ribeirão Preto, Universidade de São Paulo; 1981.

14. Forattini OP. Entomologia médica. São Paulo: Faculdade de Higiene e Saúde Publica; 1962.

15. Rachou R. Anofelinos do Brasil: comportamento das espécies vetoras da malária. Rev Bras Malariol D Trop 1958;10:145-181.

16. Logan JA. The Sardinian project: an experiment in the eradication of an indigenous malarious vector. Baltimore: The Johns Hopkins University Press; 1953. 
17. Organización Mundial de la Salud. Comité de Expertos en Paludismo: sexto informe. Ginebra: OMS; 1956. (Serie de informes técnicos 123).

18. Marques AC. Migrações internas e grandes endemias. Rev Bras Malariol D Trop 1979;31: 137-158.

19. Sabroza PC. A malária no Brasil. Cadernos Saude Publica 1985;1:71-111.

20. Shiang LS. Malária em usuários de drogas de administração endovenosa associado à soropositividade para HIV. Rev Saude Publica 1991;25:17-22.

21. Barata LCB, Andriguette MTM, Cortés MC, Meneguetti C. Surto de malária induzida em usuários de drogas injetáveis. Rev Saude Publica 1993;29:9-14.

22. Bruce-Chwatt LJ. Malaria control of the crossroads: where do we go from here? Mem Inst Oswaldo Cruz 1986;81(supl II):1-8.

23. Lepes T. Malaria: a global health problem. WHO/MAL 1981;81:749.

24. Nájera J. A global analysis of the malaria eradication in relation to the development of pri- mary health care. Geneva: World Health Organization; 1986. (WHO/MAL 86.1025).

25. Beales PF, Najera J. A reconsideration of the tactical variants for malaria control in the light of experience in their utilization. Geneva: World Health Organization; 1986. (WHO/ MAL 86.1027).

26. Orlov VS, Semashko IN. Malaria stratification as a tool in developing the strategy and tactics for modern longterm malaria control programmes. Geneva: World Health Organization; 1986. (WHO/MAL 86:1029).

27. Orlov VS, Semashko IN. Malaria stratification of territories and its practical implications. Geneva: World Health Organization; 1986 (WHO/MAL 86.1032).

28. Najera J, Liese B, Hammer JS. Health sector priorities review: malaria. Washington, DC: World Health Organization, World Bank; 1991.

29. World Health Organization. Malaria control an attainable goal: a strategy for malaria control. Trabajo presentado en la Conferencia Ministerial sobre Malaria, Reunión Interre- gional para el África, Brazzaville, Congo, 21 a 25 de octubre de 1991.

30. Greenwood BM. The microepidemiology of malaria and its importance to malaria control. Trans Royal Soc Trop Med Hyg 1989;83(supl): 25-29.

31. Organización Mundial de la Salud. Estrategia mundial de la lucha contra el paludismo. Trabajo presentado en la Conferencia Ministerial, Amsterdam, Países Bajos, 1992.

32. Bradley DJ. Malaria: whence and whither? En: Taegett GAT. Malaria: waiting for the vaccine. London: London School of Hygiene and Tropical Medicine; 1991.

Manuscrito recibido el 24 de mayo de 1995 y aceptado para publicación en versión revisada el 30 de abril de 1996.

ABSTRACT Since the 1970s, when malaria had seemed at the threshold of eradication, its incidence has increased in several countries of the world. This situation posed a series of questions, and for some time malariologists and public health authorities scarcely understood what was happening. In order to better comprehend the process, the author of this article studied the frequency of malaria cases in São Paulo, Brazil, from 1930 to 1990, attempting to examine the following factors in each of the periods studied: the importance of malaria in the society, the conditions under which the disease occurred, the epidemiologic knowledge of the time, the available technical instruments, and the control strategies that were used. Through the construction of technological models based on these factors, it became clear that the occurrence of the disease, knowledge about it, and, consequently, the ways it was dealt with changed over time. In light of this research, the paper discusses current options for the control of malaria.

Technological organization of Brazil, from 1930 to 1990 